\title{
Radiative properties of carriers in CdSe-CdS core-shell heterostructured nanocrystals of various geometries
}

S. Zhou

L. Dong

lindong@kth.se

\section{S. Popov}

\section{A. Friberg}

\author{
Division of Optics, Royal Institute of Technology, SE-164 40 Kista, Sweden \\ Division of Optics, Royal Institute of Technology, SE-164 40 Kista, Sweden \\ Division of Optics, Royal Institute of Technology, SE-164 40 Kista, Sweden \\ Division of Optics, Royal Institute of Technology, SE-164 40 Kista, Sweden \\ Department of Applied Physics, Aalto University, FI-00076 Aalto, Finland \\ Department of Physics and Mathematics, University of Eastern Finland, FI-80101 Joensuu, Finland
}

We report a model on core-shell heterostructured nanocrystals with CdSe as the core and CdS as the shell. The model is based on one-band Schrödinger equation. Three different geometries, nanodot, nanorod, and nanobone, are implemented. The carrier localization regimes with these structures are simulated, compared, and analyzed. Based on the electron and hole wave functions, the carrier overlap integral that has a great impact on stimulated emission is further investigated numerically by a novel approach. Furthermore, the relation between the nanocrystal size and electron-hole recombination energy is also examined.

[DOI: http://dx.doi.org/10.2971/jeos.2013.13042]

Keywords: CdSe-CdS core-shell nanocrystal, FEM model, carrier distribution, electron-hole recombination energy, nanobone

\section{INTRODUCTION}

Colloidal semiconductor nanocrystals (NCs), or quantum dots (QDs), have continually been an active research area which introduces a variety of applications involving photonic devices, telecommunication, information storage, medical instruments, etc $[1,2]$. Among the various formations of semiconductor NCs, core-shell heterostructured NCs earn special attention due to the feature that the carrier distribution wave function can be manipulated by varying the core/shell dimensions, which alter the NCs' physical properties significantly [3]. An important objective of wave function engineering is to separate the electrons and holes in space. Such a spatial separation of carriers triggers the quantum confined Stark effect (QCSE) which enhances single-exciton optical gain [4, 5]. QDs with carriers spatially separated are terms as being on the type-II regime. The type-II QDs show considerably lower lasing threshold than other QDs without carrier separation which need a much higher excitation energy to achieve biexciton lasing [6].

The subject of optical gain and lasing in core-shell QDs has been intensively investigated during the past decade. Light amplification and its dependence on NCs size were reported [4], followed by the realization of single-exciton optical gain [5]. In theoretical study, an approach towards analytical calculation of electronic structures of core-shell QDs was demonstrated [7], along with simulations taking into account the ambient environment of the QDs [8]. It is noted that although [8] deals only with the energy states in the conduction band, it provides a good example for boundary condition analysis and carrier distribution calculation.

The present work concentrates on heterostructured NCs with CdSe as the core and CdS as the shell. In recent studies $[9,10]$ it has been demonstrated that the core/shell combination of CdSe/CdS allows making QDs that can be flexibly tuned between type-I and type-II regimes with different core/shell sizes. The feature is termed as quasi-type-II and it is attributed to the small value of the conduction band offset with the two materials. Consequently, the NCs show potentials for both type-I applications such as light emitting devices and type-II applications like lasing.

On the other hand, variety of geometrical QD shapes available with modern chemical and nano-engineering technology, allows to develop materials for many other applications in photonics, not only to obtain optical gain and lasing. Here can be mentioned electro-optical effect, photo-refraction, birefringence and others. Simulations of such material properties using analytical approach are rather limited (if possible at all) for geometries with more complicated symmetry than spherical or simplified cylindrical, which currently attract much research interest [9]. Thus, a consistent numerical model is pertinent to thoroughly map the optical properties, most importantly the carrier separation, of the CdSe-CdS NCs over the whole range of core/shell dimensions that are technically available. 
In this work, we make a certain step ahead, and demonstrate the "prove of concept" allowing to simulate QDs of more complicated geometry. A novel approach based on Comsol Multiphysics software package is introduced to calculate both the electron and hole states in three-dimensional NC structures. The interactions between the carriers are studied to examine the dependence of the carrier overlap integral and the recombination energy on the NCs sizes. Compared to the analytical calculation in [7], the present model is more time efficient and, at the same time, it features a strong capability of processing novel and sophisticated geometries of the NCs.

At first, two regular structures, nanodot and nanorod, are introduced. We report a result on carrier distribution in nanorods that differs from that of [3]. However, our result matches the results of [7], [11], and [12]. Subsequently, a novel nanobone structure is proposed that has interesting properties of zero overlap integral and a carrier localization shift. A relatively efficient and reliable method of modelling the CdSe-CdS NC structures and related quantum mechanical problems is demonstrated.

\section{QUANTUM MECHANICS BACKGROUND}

As one of the building blocks of Comsol modelling, the quantum theory that deals with the behaviour of carriers confined in heterostructured NCs is discussed briefly in this section. The starting point is the one-band Schrödinger equation using effective mass approximation

$$
\left[-\frac{\hbar^{2}}{2 m_{a}^{*}(r)} \nabla^{2}+V_{a}(r)\right] \psi_{a}(r)=E_{a} \psi_{a}(r)
$$

where $a$ stands for the electron or hole, $\hbar$ is the reduced Planck constant, and $m_{a}^{*}(r), V_{a}(r), E_{a}, \psi_{a}(r)$ are the positiondependent effective mass, position-dependent band edge potential, eigen energy, and position-dependent carrier envelop function, respectively, for an electron or a hole. From the point of view of energy conservation, the first term on the left-hand side represents particle kinetic energy, while the second term represents the confinement energy. The right-hand side term is the total carrier energy. Eq. (1) is solved numerically to study the carrier dynamics in semiconductor NCs. The details will be discussed in the later section.

For a core-shell heterostructured system embedded in a dielectric polymer environment, a discontinuity of the conduction and valence band gap exists at the core-shell interface (internal boundary) and also at the interface between the shell and the polymer (external boundary). The confinement potential energy can be represented as

$$
V(r)= \begin{cases}V_{c} & r<R \\ V_{s} & R<r<R+H \\ \infty & r>R+H\end{cases}
$$

where $R$ is the core radius and $H$ is the shell thickness.

For the interior boundary between the core and shell, one imposes the BenDaniel-Duke condition [13]

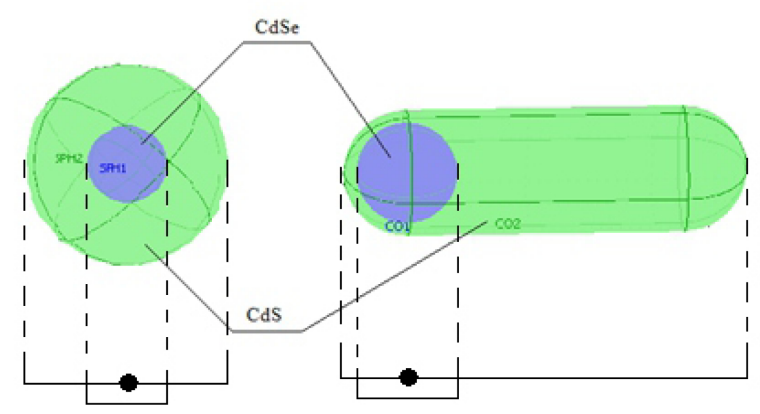

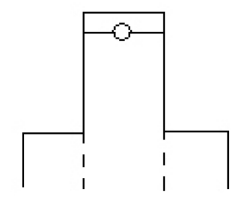

(a)

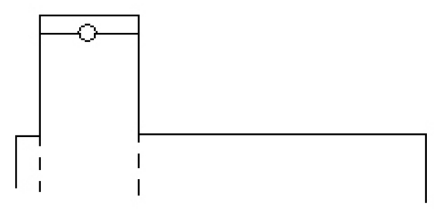

(b)
FIG. 1 (a) Nanodot and (b) nanorod with their bandstructures. The black dot is an electron and the white dot is a hole.

$$
\begin{array}{r}
\psi_{c}(r)=\psi_{s}(r) \\
\frac{1}{m_{a}^{*}(r)} \nabla \psi_{c}(r)=\frac{1}{m_{a}^{*}(r)} \nabla \psi_{s}(r)
\end{array}
$$

for the envelope function; it applies to carrier transposition across a heterojunction interface. Indices " $\mathrm{c}$ " and " $\mathrm{s}$ " are attributed to the core and the shell, respectively.

\section{MODEL CONSTRUCTION}

Before approaching the solution of Eq. (1), we first examine the modelling process in Comsol. In this case, one starts from defining the geometry corresponding to core/shell heterstructured NCs as shown in Figure 1 for a nanodot and a nanorod. Both 3D geometries represent the virtual images of quantum dots. For the samples in this work, CdSe and CdS are supposed to be filled in the core and shell, respectively, which forms a CdSe/CdS heterostructure that, for instance, can be used as the basis of photovoltaic devices. Then we assume that the QDs are embedded in a polymer PMMA environment where individual QDs are grown separately enough so that the carrier wave functions do not overlap and interfere with each other in the global structure.

We next consider some specific formalities to clarify the physics of the situation in $3 \mathrm{D}$ geometry. To solve a quantum mechanics problem in Comsol, we apply the Eigen-value analysis module which introduces the standard eigen-value Partial Differential Equation (PDE) coefficient form

$$
\nabla(-c \nabla u-\alpha u+\gamma)+a u+\beta \nabla u=d_{a} \lambda u-e_{a} \lambda^{2} u
$$

By comparing Eqs. (1) and (4), one obtains the coefficient settings summarized in Table 1.

The conduction band offset at the CdSe/CdS interface is one of the critical parameters for the simulation. It is by far not 


\begin{tabular}{ccccccccc}
\hline$u$ & $c$ & $a$ & $\beta$ & $d_{a}$ & $e_{a}$ & $\alpha$ & $\gamma$ & $\lambda$ \\
\hline$\psi_{a}$ & $\frac{\hbar^{2}}{2 m_{a}^{*}}$ & $V_{a}$ & 0 & 1 & 0 & 0 & 0 & $E_{a}$ \\
\hline
\end{tabular}

TABLE 1 PDE coefficient settings

well established among the researchers. The reported values for the offset vary from -0.3 to $+0.3 \mathrm{eV}$ [14]-[16]. Nevertheless, compared to the valence band offset of $0.78 \mathrm{eV}$, all these values are relatively small in terms of carrier confinement, so that the choice among the different values does not influence the simulation results of electron distribution very much [17]. In this work, the value of $-0.1 \mathrm{eV}$ is chosen. In such a case, a heterojunction with a straddling energy gap is formed (Figure 1). The important parameters for the sub-domain settings are listed in Table 2.

\begin{tabular}{ccccc}
\hline Parameter & $m_{e}^{*}\left(m_{0}\right)$ & $m_{h}^{*}\left(m_{0}\right)$ & $V_{e}(e V)$ & $V_{h}(e V)$ \\
\hline$C d S e$ & 0.13 & 0.45 & 0 & 0 \\
$C d S$ & 0.2 & 0.7 & $-0.1^{* *}$ & 0.78 \\
\hline
\end{tabular}

TABLE 2 Effective mass and band edge potentials for CdSe and CdS, **The sign is changed to positive in sub-domain settings due to the negative charge of an electron.

To implement the boundary settings, the relevant coefficients $q, g, r$, and $h$ are included in the generalized von Neumann condition

$$
\mathbf{n} \cdot\left((c \nabla u+\alpha u-\gamma)_{1}-(c \nabla u+\alpha u-\gamma)_{2}\right)+q u=g
$$

and in the Dirichlet boundary condition

$$
\begin{aligned}
& \mathbf{n} \cdot\left((c \nabla u+\alpha u-\gamma)_{1}-(c \nabla u+\alpha u-\gamma)_{2}\right)+q u \\
& \quad=g-h^{T} \mu \\
& h u=r
\end{aligned}
$$

where $u$ corresponds to the envelope function $\psi$ in Eq. (1) and $h^{T}$ is the transpose of $h$ in a matrix form. The coefficients for both the external and internal boundaries can be determined by considering the physical meaning on each boundary:

External boundary. Since the NCs are surrounded by polymer, the carrier wave functions cease at the external boundary. Thus they satisfy the Dirichlet condition for which $h=1$ and $r=0$, when $u$ is zero. Inserting this result into Eqs. (5) and (6a) leads to $q=g=0$. Therefore, one may simply ignore Eq. (5) for external boundaries.

Internal boundary. The quantity $\Gamma=-c \nabla u-\alpha u+\gamma$ is called the flux vector, which can experience a discontinuity in its normal component across the internal boundary. Denoting the two adjacent sub-domains as 1 and 2 (Figure 1), Eq. (6a) can be rewritten as

$$
-\mathbf{n}_{\mathbf{1}} \cdot \boldsymbol{\Gamma}_{\mathbf{1}}-\mathbf{n}_{\mathbf{2}} \cdot \boldsymbol{\Gamma}_{\mathbf{2}}+q u=g-h^{T} \mu
$$

which describes the jump of the normal component of $\Gamma$ across the boundary. As previously described, the BenDaniel-Duke conditions ( $3 a$ ) and ( $3 b$ ) are imposed at the internal boundary. By multiplying both sides of Eq. (3b) by $\hbar^{2} / 2$, one gets

$$
c_{1} \nabla u_{1}=c_{2} \nabla u_{2}
$$

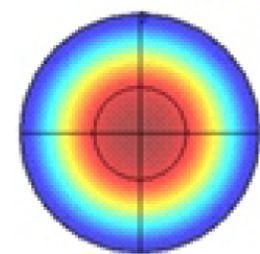

(a)

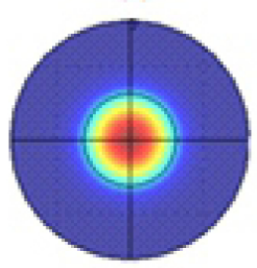

(c)

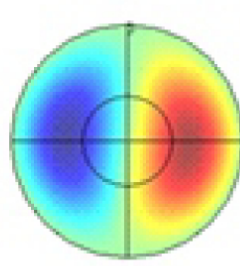

(b)

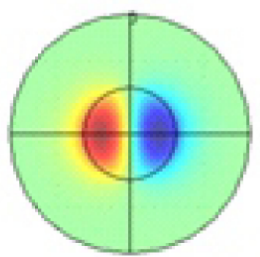

(d)

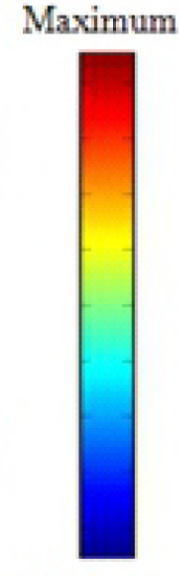

Minimum
FIG. 2 Cross section schematics of carrier wave function $\psi_{a}$ for (a) first and (b) second order electrons, along with (c) first and (d) second order holes in spherical NCs of cdse-cds.

Since $\alpha=\gamma=0$ and $n_{1}=-n_{2}$, the following relation is obtained

$$
-\mathrm{n}_{1} \cdot \Gamma_{1}=\mathrm{n}_{2} \cdot \Gamma_{2}
$$

On inserting Eq. (9) into Eqs. (5), (6a) and (6b), we find that all the coefficients $q, g, r$, and $h$ are zero. All the coefficients for the boundary settings are summarized in Table 3.

\begin{tabular}{ccc}
\hline & $q, g, r$ & $h$ \\
\hline External boundary & 0 & 1 \\
\hline Internal boundary & 0 & 0 \\
\hline
\end{tabular}

TABLE 3 Boundary conditions

\section{NUMERICAL RESULTS AND DISCUSSION}

In this section, we demonstrate the modeling results followed by some discussions.

The first and second order solutions of the electron wave function $\psi_{e}$ for spherical CdSe-CdS NCs are shown in Figures 2(a) and 2(b), respectively. The core radius is $1.25 \mathrm{~nm}$ and the shell thickness is $2 \mathrm{~nm}$. The maximum and minimum values in the scale bar are 2.177 and 0 (in arbitrary units) for Figure 2(a), and 2.274 and -2.274 for Figure 2(b). The electron eigen-energies for Figures 2(a) and 2(b) are $0.104 \mathrm{eV}$ and $0.298 \mathrm{eV}$, respectively. It is observed that due to the small value of the conduction band offset the electrons are spreading from the core into the shell. For the first order solution, the probability of finding electrons in the core is higher than that in the shell. For the second order solution, two symmetrical localization centers are found in the shell region.

The first and second order solutions of the wave function $\psi_{h}$ for the holes are shown in Figures 2(c) and 2(d). The maximum and minimum values in the scale bar are 7.017 and 0 for Figure 2(c), and 6.792 and -6.792 for Figure 2(d). The hole eigen- 


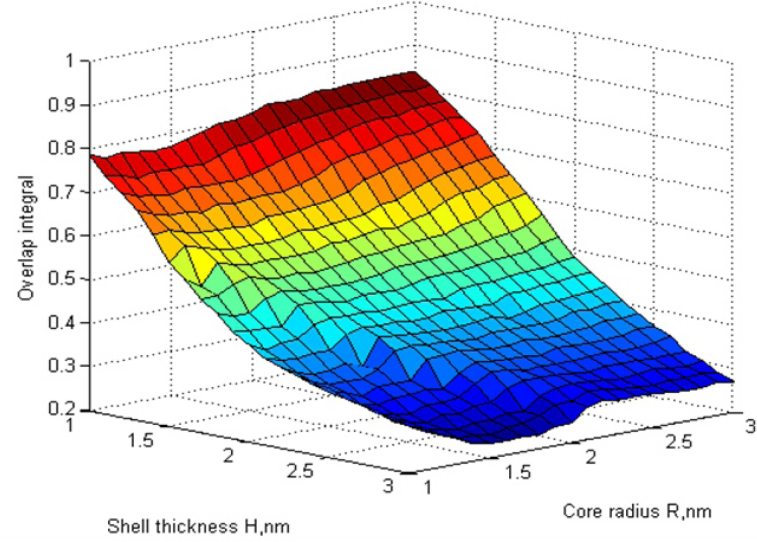

FIG. 3 Carrier overlap integral as a function of core radius and shell thickness for cdse-cds nanodots.

energies for Figures. 2(c) and 2(d) are $0.289 \mathrm{eV}$ and $0.576 \mathrm{eV}$, respectively. It should be pointed out that due to the large valence band offset, the holes are well confined in the CdSe core for both orders of solutions. The electron-hole overlap integral, defined as

$$
\Theta_{e h}=\left|\left\langle\psi_{h} \mid \psi_{e}\right\rangle\right|^{2}=\left[\int \psi_{h}^{*} \psi_{e} d V\right]^{2}
$$

is a critical parameter in wave function engineering in heterostructured NCs since it indicates the degree of carrier separation and exciton-exciton interaction energy [5]. A reduction in $\Theta_{e h}$ leads to an increase of the electric field intensity by the Stark shift and to a decrease of the radiative decay rate of the excitons [3], bringing a larger probability of population inversion. The overlap integral is closely related to the sizes of the core and shell in the NCs [18]. Since the wave functions of both electrons and holes are found in explicit form, it is convenient to calculate the overlap integral of the carriers.

The calculation results of the carriers' overlap integral for spherical CdSe-CdS core-shell QDs are shown in Figure 3 as a function of the core radius and the shell thickness in technology affordable range (1-3 nm). It is noted that the integral value is more affected by the shell thickness than the core radius. The thickening of the shell can effectively extract the electrons out of the core. The minimum value of the overlap integral is 0.23 with core radius of $1.8 \mathrm{~nm}$ and shell thickness of $3 \mathrm{~nm}$. On the other hand, the maximum value exceeds 0.8 with a large core and thin shell. The quasi-type-II feature of the dots is confirmed with the large span of the electron-hole separation. It indicates the potential of the QDs for diverse applications, such as light source with high emission intensity, or gain media for lasing.

One of the problems counteracting building up stimulated emission (to realize quantum dot lasers) is the non-radiative Auger recombination which leads to an ultrafast recombination rate of the excitons and drastically drains the optical gain. Separating the electrons and holes by wave function engineering is expected to allow lasing in the single-exciton regime where Auger recombination is inactive [5], so that the lasing threshold can be significantly reduced. The single-exciton recombination energy is calculated with the model via the rela-

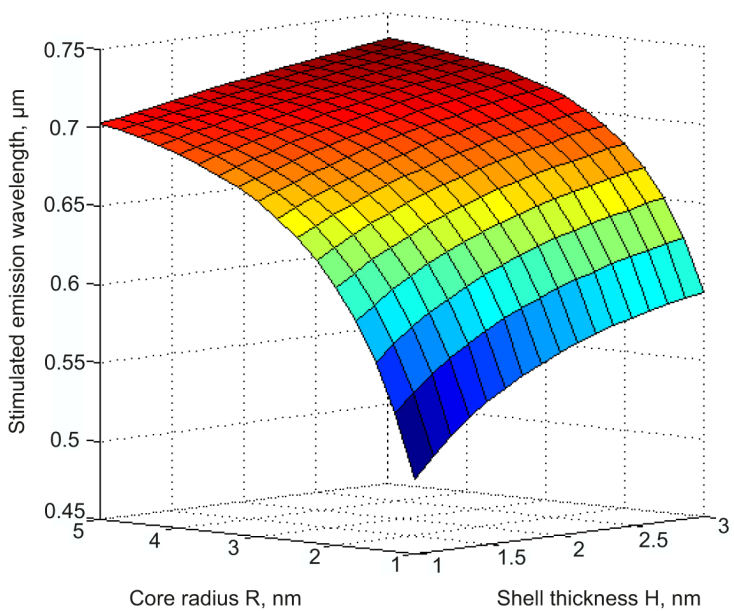

FIG. 4 Stimulated emission wavelength of spherical CdSe-CdS nanodots with respect to the core radius and shell thickness.

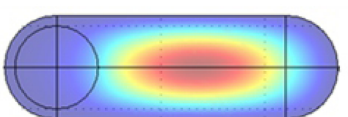

(a)

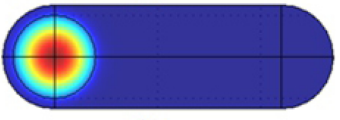

(c)

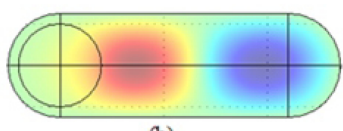

(b)

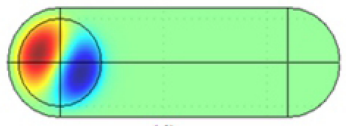

(d)

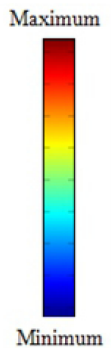

FIG. 5 Cross section schematics of carrier wave function $\psi_{a}$ for (a) first and (b) second order electrons, along with (c) first and (d) second order holes in CdSe-CdS nanorods.

tion

$$
E_{x}=E_{g}+E^{e}+E^{h}+W^{e h}+U^{e h}+V^{e}+V^{h}
$$

In $(11), E_{g}$ is the gap between the minimum of the conduction band and maximum of the valence one, while $E^{e}$ and $E^{h}$ correspond to the eigen-energies of the electrons and holes. Additionally, $W^{e h}, U^{e h}, V^{e}$, and $V^{h}$ represent the Coulomb coupling interaction, interface polarization energy, and electron/hole dielectric solvation energy, respectively. It is found in the calculation that only the first three terms on the right side of Eq. (11) dominate. The stimulated emission wavelengths of the first order carrier recombination for spherical CdSe-CdS nanodots are shown in Figure 4.

It is known that the carrier separation with spherical NCs is not straightforward. The Gaussian distribution of the electrons determines that they cannot be fully extracted to the shell. Thus, it is impossible to totally eliminate the rapid recombination of both electrons and holes within the core. Meanwhile, the sensitive dependence of the overlap integral on the core/shell dimensions brings difficulties to the fabrication in terms of size distribution control. Therefore, the potential of the dots to achieve optical gain is restricted. A high aspect ratio nanorod is one of the attractive approaches to overcome the limitation. The distribution maximum of electrons can be moved from the core into the elongated shell, meanwhile with the holes still being confined in the core. In this case, a more thorough carrier separation can be achieved. 


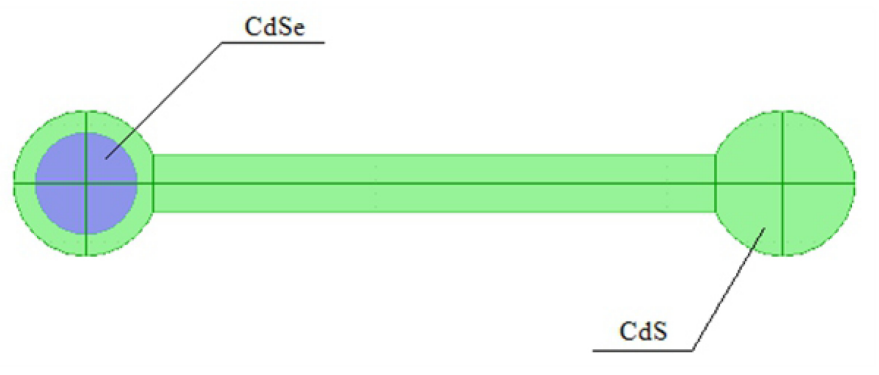

FIG. 6 Schematic of core-shell bone structure.

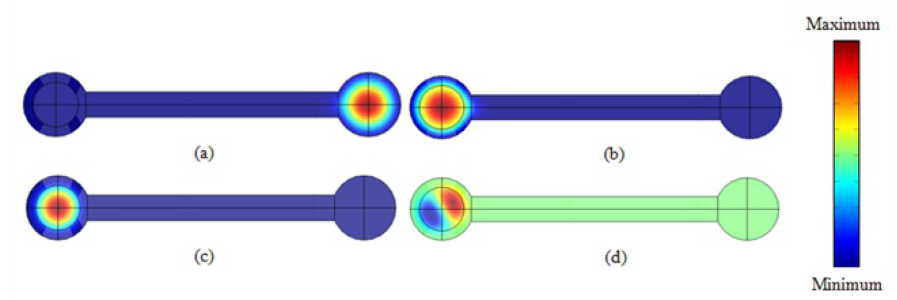

FIG. 7 Cross section schematics of carrier wave function $\psi_{a}$ for (a) first and (b) second order electrons, along with (c) first and (d) second order holes in bone-like CdSe-CdS NCS

The simulation results of carrier distribution within CdSe-CdS core/shell nanorod are shown in Figure 5. The core radius is $2 \mathrm{~nm}$ and the shell length is $16 \mathrm{~nm}$. The maximum and minimum values in the scale bar are 3.678 and 0 for Figure 5(a), and 3.403 and -3.42 for Figure 5(b). The electron eigen-energies for Figures 5(a) and 5(b) are $0.089 \mathrm{eV}$ and $0.126 \mathrm{eV}$, respectively. The maximum and minimum values in the scale bar are 5.35 and 0 for Figure 5(c), and 4.378 and -3.42 for Figure 5(d). The hole eigen-energies for Figure 5(c) and (d) are $0.143 \mathrm{eV}$ and $0.293 \mathrm{eV}$, respectively. It is noticed that with both the first and second order solutions the carriers are completely separated in space within the nanorods, which is promising to introduce QCSE to further encourage single-exciton optical gain.

Furthermore, a novel bone-like heterostructure is proposed and investigated (Figure 6). The core/shell materials are adopted from the nanodot and nanorod. The spheres at both ends are of the same size while the CdSe core is located only at one end. A core diameter of $3.5 \mathrm{~nm}$ and a shell thickness of $1.5 \mathrm{~nm}$ are implemented in the simulation. The middle link is $20 \mathrm{~nm}$ long and $2 \mathrm{~nm}$ wide.

The first and second order solutions of the electron wave function $\psi_{e}$ for the nanobone are shown in Figures 7(a) and $7(b)$, respectively. The maximum and minimum values in the scale bar are 10.117 and 0 for Figure 7(a), and 5.463 and 0 for Figure 7(b). The electron eigen-energies for Figures 7(a) and 7(b) are $0.300 \mathrm{eV}$ and $0.339 \mathrm{eV}$, respectively. The first and second order solutions of the wave function $\psi_{h}$ for the holes are shown in Figures 7(c) and 7(d). The maximum and minimum values in the scale bar are 8.823 and 0 for Figure 7 (c), and 6.373 and -6.405 for Figure $7(\mathrm{~d})$. The hole eigen-energies for Figures $7(\mathrm{c})$ and $7(\mathrm{~d})$ are $0.176 \mathrm{eV}$ and $0.359 \mathrm{eV}$, respectively.

For the first order solution, the electrons and the holes are fully separated into the two ends of the structure, which is

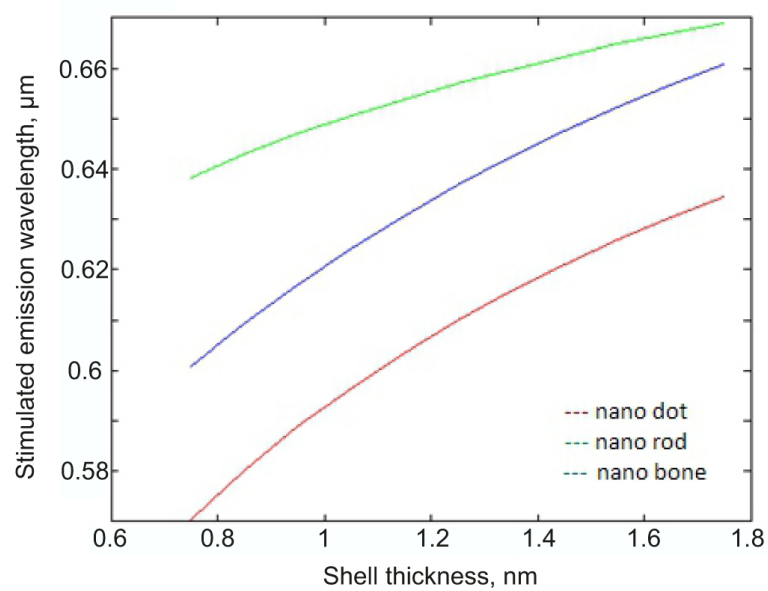

FIC. 8 Stimulated emission wavelength of CdSe-CdS nanospheres, nanorods, and nanobones with core radius of $1.75 \mathrm{~nm}$ and shell thickness from 0.75 to $1.75 \mathrm{~nm}$.

of great importance for achieving single-exciton optical gain. Interestingly, the electrons are "jumping" back from the noncore end to the core end for the second order solution, while the holes are still confined in the core. Such a peculiar behavior can have a fundamental origin related to rather complicated distribution of energy potential through the nano-bone structure and different effective masses of electrons and holes. Due to smaller effective mass (as a general case), electrons tend to easier pass a "bottleneck" bridge between the two ends and occupy a state with lower energy, that is not the case for holes with lower mobility. However, to fully understand the phenomenon, much more detailed investigations are required.

The strong contrast of the overlap integral for the first two orders of solutions (including most of the carriers) suggests that the bone-like NCs may be suitable not only for lasing applications, but also for situations in which logical or intelligent optical components are involved. For example, clear spatial separation of electron-hole pairs can be implemented to generate a photo-refractive effect. Strong polarization response of elementary dipoles (here nano-bones can be considered as "macro-dipoles") induced by optical excitation results in modulation of refractive index, that can be used to create an elementary optical gate.

The stimulated emission wavelength of the nanobone is examined. The results show that the wavelength does not depend on the length of the middle link in the structure. This implies that the nanobone structure has a high tolerance for its length variation when it is grown for lasing applications.

In Figure 8, the stimulated emission wavelengths of CdSeCdS nanodots, nanorods, and nanobones are compared. All three structures have the same core radius of $1.75 \mathrm{~nm}$. The shell thickness varies from 0.75 to $1.75 \mathrm{~nm}$. It is seen that with all the structures the stimulated emission is red shifted as the shell thickness increases, which is attributed to the size effect of QDs. With equal core radius and shell thickness, the nanorod presents the longest stimulated emission wavelength, followed by the nanobone and the nanodot. 


\section{CONCLUSIONS}

A novel model based on the one-band Schrödinger equation is proposed for CdSe-CdS core-shell heterostructured nanocrystals. The carrier distribution, separation, and eigen-state energies can be determined numerically with the model. Different geometries of nanodot, nanorod, and nanobone are investigated. Indeed, this approach can be used practically for any 3D geometry of QDs (sophisticate tetrahedral shapes shown in [9] is one of the examples) that is impossible with analytical methods. This can be also very useful for analysing possible deviation of the optical properties of QDs due to fabrication imperfections resulting in distortions of originally designed shapes. The quasi-type-II feature of CdSe-CdS nanodots is confirmed by mapping the carrier overlap integral on varying core/shell sizes. The proposed bone-like structures show distinct features of carrier distribution, which are attractive for novel photonic applications. Additionally, the model is advantageous in its time efficiency and capability of solving NCs with irregular and sophisticated geometries.

\section{ACKN OWLEDGEMENTS}

We acknowledge the Academy of Finland and ADOPT- Linné center of advanced Optics and Photonics (Sweden) for their financial support.

\section{References}

[1] 0. Svelto, D. Hanna, Principle of Laser Engineering (Plenum Publishers, New York, 1998).

[2] V. Klimov, Nanocrystal Quantum Dots (CRC Press, Boca Raton, 2010).

[3] J. Müller, J. M. Lupton, P. Lagoudakis, F. Schindler, R. Koeppe, A. Rogach, and J. Feldmann, "Wave function engineering in elongated semiconductor nanocrystals with heterogeneous carrier confinement," Nano Lett. 5, 2044-2049 (2005).

[4] S. Ivanov, J. Nanda, A. Piryatinski, M. Achermann, L. Balet, I. Bezel, P. Anikeeva, et al., "Light amplification using inverted core/shell nanocrystals: towards lasing in the single-exciton regime," J. Phys. Chem. B 108, 10625-10630 (2004).

[5] V. Klimov, S. Ivanov, J. Nanda, M. Achermann, I. Bezel, J. McGuire, and A. Piryatinski, "Single-exciton optical gain in semiconductor nanocrystals," Nature 447, 441-446 (2007).
[6] V. Klimov, A. Mikhailovsky, S. Xu, A. Malko, J. Hollingsworth, C. Leatherdale, H. Eisler, and M. Bawendi, "Optical gain and stimulated emission in nanocrystal quantum dots," Science 290, 314-317 (2000).

[7] Y. Luo, and L. Wang, "Electronic structures of the CdSe/CdS coreshell nanorods," ACS Nano 4, 91-98 (2010).

[8] R. Melnik, and M. Willatzen, "Bandstructures of conical quantum dots with wetting layers," Nanotechnology 15, 1-8 (2004).

[9] A. Sugunan, Y. Zhao, S. Mitra, L. Dong, S. Li, S. Popov, S. Marcinkevicius, et al., "Synthesis of tetrahedral quasi-type-II CdSe-CdS coreshell quantum dots," Nanotechnology 22, 425202 (2011).

[10] L. Dong, A. Sugunan, J. Hu, S. Zhou, S. Li, S. Popov, M. Toprak, et al., "Photoluminescence from quasi-type-Il spherical CdSe-CdS core-shell quantum dots," Appl. Optics 52, 105-109 (2013).

[11] M. Lupo, F. Sala, L. Carbone, M. Zavelani-Rossi, A. Fiore, L. Luer, D. Polli, et al., "Ultrafast electron-hole dynamics in core/shell CdSe/CdS dot/rod nanocrystals," Nano Lett. 8, 4582-4587 (2008).

[12] A. Rogach, T. Klar, J. Lupton, A. Meijerinkd, and J. Feldmanna, "Energy transfer with semiconductor nanocrystals," J. Mater. Chem. 19, 1208-1221 (2009).

[13] D. BenDaniel, and C. Duke, "Space-charge effects on electron tunneling," Phys. Rev. 152, 683-692 (1966).

[14] A. Nethercot, "Prediction of Fermi energies and photoelectric thresholds based on electronegativity concepts," Phys. Rev. Lett. 33, 1088-1091 (1974).

[15] C. Trager-Cowan, P. Parbrook, B. Henderson, and K. O'Donnell, "Band alignments in $\mathrm{Zn}(\mathrm{Cd}) \mathrm{S}(\mathrm{Se})$ strained layer superlattices," Semicond. Sci. Tech. 7, 536-541 (1992).

[16] K. O'Donnell, P. Parbrook, F. Yang, X. Chen, D. Irvine, C. TragerCowan, B. Henderson, et al., "The optical properties of wide bandgap binary II-VI superlattices," J. Cryst. Growth 117, 497-500 (1992).

[17] X. Peng, M. Schlamp, A. Kadavanich, and A. Alivisatos, "Epitaxial growth of highly luminescent $\mathrm{CdSe} / \mathrm{CdS}$ core/shell nanocrystals with photostability and electronic accessibility," J. Am. Chem. Soc. 119, 7019-7029 (1997).

[18] J. Haus, H. Zhou, I. Honma, and H. Komiyama, "Quantum confinement in semiconductor heterostructure nanometer-size particles," Phys. Rev. B 47, 1359-1365 (1993). 